Sabine Ammon, Eva Maria Froschauer

\title{
Zur Einleitung: Wissenschaft Entwerfen. Perspektiven einer reflexiven Entwurfsforschung
}

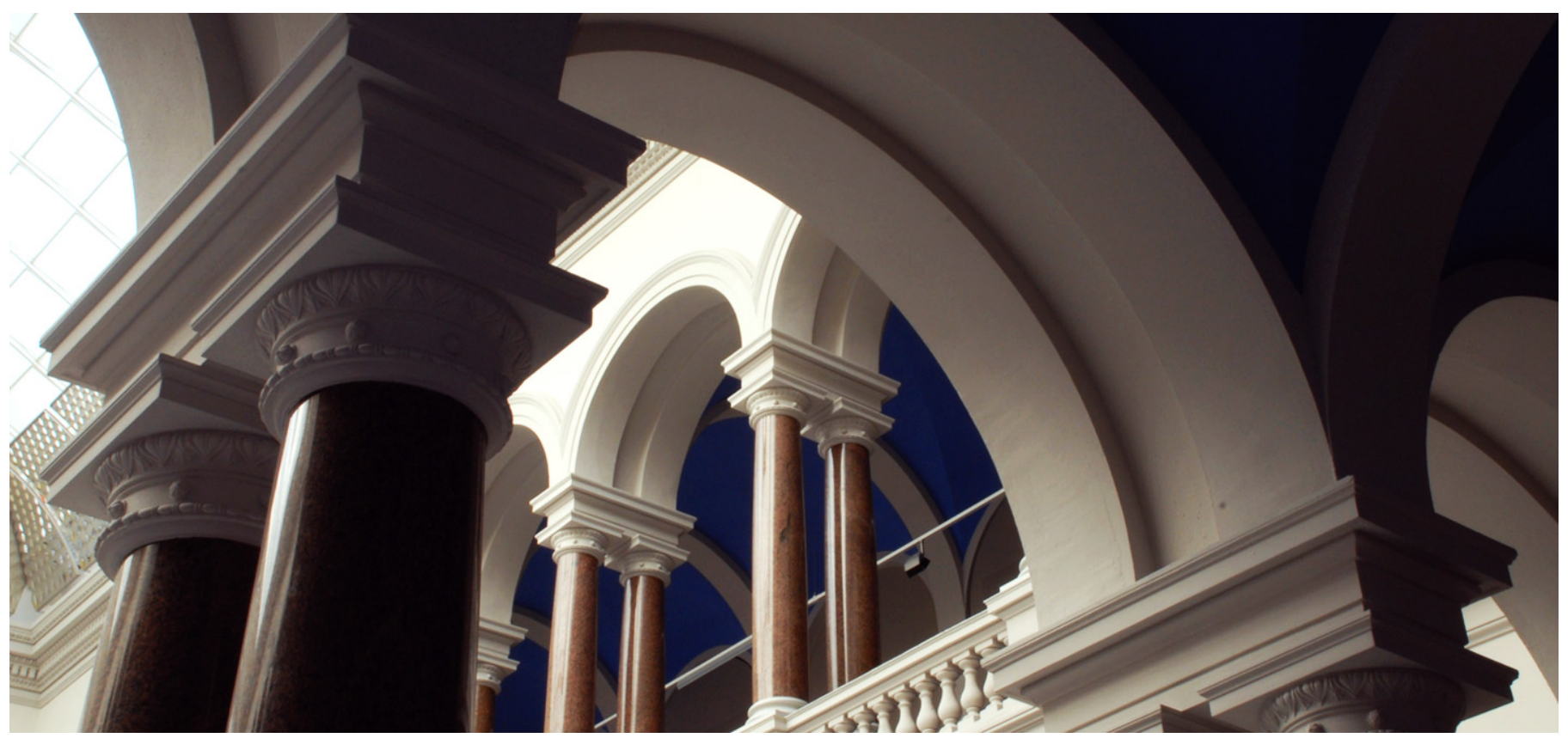

\section{Suggested Citation}

Ammon, Sabine; Froschauer, Eva Maria: Zur Einleitung: Wissenschaft Entwerfen. Perspektiven einer reflexiven Entwurfsforschung. - In: Ammon, S.; Froschauer, E. (Hrsg.): Wissenschaft entwerfen. Vom forschenden Entwerfen zur Entwurfsforschung der Architektur. - Paderborn: Fink, 2013. - ISBN: 978-3-7705-5521-5. - S. 15-38. 


\section{Zur Einleitung: \\ Wissenschaft Entwerfen. \\ Perspektiven einer \\ reflexiven Entwurfsforschung}

Sabine Ammon, Eva Maria Froschauer

Das Entwerfen nimmt im Selbstverständnis der Architektur eine zentrale Rolle ein: Es gilt für Theorie wie Praxis als wesentlicher Bezugspunkt der Disziplin, welcher vor dem Hintergrund aktueller Entwicklungen drängende Fragen aufwirft. $\mathrm{Zu}$ beobachten sind die zunehmenden Bestrebungen, dem Entwerfen den Status des Forschens zu geben und es auf die Ebene der Wissenschaftlichkeit zu heben. Zugleich herrscht Unsicherheit darüber, wie sich die komplexe Tätigkeit des Entwurfshandelns methodisch erschließen und systematischer Forschungsarbeit zugänglich machen lässt. Diese Situation nimmt der vorliegende Band zum Anlass, die Frage nach dem Entwerfen als Wissenschaft neu zu stellen. Im Vordergrund steht dabei die übergreifende Untersuchung des Gegenstands, die auf ein besseres Verständnis des Entwerfens zielt. Am Beispiel des Architekturentwurfs werden neben praxisorientierten Überlegungen systematische und historische Betrachtungen unterschiedlicher Disziplinen zusammengeführt, um den Bedingungen und Möglichkeiten einer genuinen `Entwurfsforschung nachzuspüren. Dabei geht es weniger darum, bereits fertige Antworten zu geben, als vielmehr die derzeitige Diskussion um wichtige Facetten zu erweitern. 
Das Spannungsfeld zwischen >Wissenschaft $<$ und `Entwerfen verweist auf einen großen Fragenkomplex, der-um das Offensichtliche gleich vorwegzunehmen-nicht umfassend im Rahmen dieses Bandes beantwortet werden kann. Wer nach der Verhältnisbestimmung von Wissenschaft und Entwerfen fragt, hat bereits eine wichtige Grundannahme getroffen: Das Entwerfen wird nicht nur als Vorgang betrachtet, der neue Artefakte schafft, sondern es wird zugleich suggeriert, dass das Entwerfen auch als Erkenntnisprozess gesehen werden muss, der Wissen hervorbringt. Wäre dem nicht so, könnte keine Lehre oder Vermittlung von Entwurfshandlungen erfolgen, stellten gestalterische Ausbildung und Studienabschlüsse keine nachvollziehbaren Unterfangen dar. Auch gälten Versuche der stärkeren Systematisierung, Standardisierung oder Methodologisierung von vornherein als aussichtslos. Doch impliziert die Annahme eines Wissens, das aus dem Entwurfsvorgang hervorgeht, nicht unmittelbar eine Entwurfswissenschaft. Praktiken der Absicherung und Überprüfung, der Reflexion und Abstraktion müssen erst hinzukommen, um aus dem Entwurfswissen eine Wissenschaft werden zu lassen.

Wenn sich nun der vorliegende Band das Ziel gesteckt hat, nach den Perspektiven aktueller Entwurfsforschung zu fragen, konzentriert sich dies auf einen wichtigen Ausschnitt: Im Zentrum steht eine reflexive Entwurfsforschung, die sich um ein besseres Verständnis des Entwerfens bemüht. Sie interessiert sich dabei weniger für die späteren Artefakte als Produkte dieses Vorgangs als vielmehr für die Analyse und Beschreibung der Prozesse, ohne in sie selbst einzugreifen. Dadurch setzt sich die reflexive Entwurfsforschung einerseits von einer angewandten Entwurfsforschung ab, die auf optimierte Produktentwicklungen zielt, andererseits von der aktuell viel diskutierten praktischen Entwurfsforschung, die den Entwurfsvorgang selbst zur empirischen Forschungsmethode erhebt. Statt auf eine Forschung `durch das Entwerfen konzentriert sich der Band vielmehr auf eine Forschung >über` das Entwerfen. ${ }^{1}$ Das dadurch erreichte, tiefere Verständnis des Entwurfsvorgangs kann nicht nur zu einer reflexiven Praxis in Lehre und Berufsalltag beitragen und die Entwurfsforschung sinnvoll ergänzen, ${ }^{2}$ sondern es kann auch zu einer größeren Vertrautheit mit einer Kulturtechnik beitragen, die außerhalb der gestaltenden Disziplinen noch viel zu wenig zur Kenntnis genommen wird.

\section{Neubestimmung der Entwurfsforschung}

Das Bedürfnis, das Entwerfen in seinem Verhältnis zur Wissenschaft zu bestimmen, ist nicht neu-doch stellt es sich momentan 
mit neuer Dringlichkeit. Ein Rückblick auf die Entwicklungen des 19. und 20. Jahrhunderts zeigt, dass es gerade in der Architektur immer wieder einflussreiche Protagonisten und Bewegungen gegeben hat, die eine Verwissenschaftlichung des Entwerfens eingefordert oder initiiert haben. Wieder scheint es an der Zeit zu sein, nach der lange währenden Betonung des sunzugänglichen`, künstlerisch-kreativen Moments im Entwurfsvorgang, dessen implizite Methoden und Verfahren zu hinterfragen. Eine Reihe von Faktoren, die den Debatten Nahrung gegeben haben, wirken hier zusammen. Nicht unerheblich ist in dieser Hinsicht sicherlich der hochschulpolitische Anstoß, der mit der durch die Bologna-Reform ausgelösten Umgestaltung von Studiengängen sowie finanzieller Anreizsysteme für Hochschulen und Fakultäten dazu führt, dass sich nun auch die gestaltenden Disziplinen verstärkt der Forschung widmen. Der Entwurfsprozess und dessen Verortung im Kanon der Wissenschaften ist nicht nur für die Curricula, sondern insbesondere für die Konzeption der Promotionsprogramme eine Herausforderung. Auf dem Prüfstand stehen damit Formen und Inhalte der Entwurfslehren selbst, die momentan eine neue 'Selbstvergewisserung` erfahren.

Der mittlerweile deutliche zeitliche Abstand zur letzten Welle der Verwissenschaftlichung lässt Raum für eine Neubewertung: Nach dem Design Methods Movement der 1960er und frühen 1970er Jahre entwickelte sich die Entwurfsmethodologie für vier Jahrzehnte zu einem tabuisierten Thema der Architektur. Im Zuge der beginnenden historischen Aufarbeitung der damaligen Schlüsselthemen der Kybernetik und des Strukturalismus' geraten die Formalisierungs- und Systematisierungsversuche der Entwurfsmethodologen wieder ins Blickfeld, deren Erbe heute mit frischem Interesse erkundet wird. Darüber hinaus ist die Entwicklung der Informationstechnologie gegenwärtig an einen Punkt gelangt, an dem sich eine Kopplung bestimmter Aspekte der Verwissenschaftlichung mit digitalen Entwurfsmethodologien als notwendig erweist. Die überzogenen Ansprüche des Design Methods Movement, die auf eine Formalisierung und Automatisierung der Entwurfsvorgänge zielten, sind dem pragmatischen Anliegen gewichen, intelligente Werkzeuge zu entwickeln, die den Entwurfsvorgang sinnvoll unterstützen können. Hatten die anfänglichen CAAD-Werkzeuge lediglich imitierenden Charakter, um den Zeichenvorgang auf Papier nachzubilden, wurden diese später von Programmen abgelöst, die eine Arbeit mit Volumen und Bauteilen ermöglichten. Die aktuellen Software-Entwicklungen bemühen sich nun um die Einbettung entwurflicher 
Entscheidungsprozesse und Lösungsstrategien vor dem Hintergrund größer werdender Informationsmengen. Solche übergreifenden systemischen und parameterorientierten Anforderungen werfen für die Software-Programmierung Fragen auf, die an frühere Formen der Verwissenschaftlichung anknüpfen können.

Schließlich trägt auch die Neujustierung des Wissensbegriffs zu einer veränderten Betrachtung des Verhältnisses von Wissenschaft und Entwerfen bei. Nicht zuletzt durch die Pluralismustheorien der Postmoderne provoziert, vollzog sich in Wissenschaftsphilosophie und -soziologie in den letzten zwei Jahrzehnten eine Wende: Das stereotype Bild der Wissenschaften-lange geprägt von den Naturwissenschaften, allen voran die Physik als implizite Leitdisziplin-ist im Begriff, sich zu wandeln. Dabei geraten Wissensformen in den Fokus, die sich bislang einer Einordnung in klassische Kategorien entzogen haben. Dies betrifft sowohl das Alltags- oder Praxiswissen als auch jene Bereiche, die vormals als unwissenschaftlich galten oder deren gesonderte Betrachtung durch ihren Anwendungsbezug als überflüssig erachtet wurden. In der Folge erfahren die Künste ebenso wie das Ingenieurwesen und die Architektur eine wissenstheoretische Aufwertung, deren Bedingungen zunehmend Gegenstand wissenstheoretischer Studien werden. Geschaffen wird eine neue Ebene des Vergleichs, die fundierte Rückschlüsse auf Gemeinsamkeiten zulässt-aber auch Unterschiede in den Wissenspraxen klarer hervortreten lässt.

Dessen ungeachtet stellt das Entwerfen eine Herausforderung für jede Forschung dar. Denn es ist geprägt von großen Spannungsfeldern, changiert es doch zwischen regelgeleitetem Vorgehen und dessen beständigen Überschreiten, enthält es Unschärfen und unterliegt doch dem Zwang zu Präzision und Explizierung, entwickelt es sich aus spontanen Eingebungen und erfordert gleichermaßen systematisches Ausarbeiten. Bei näherer Betrachtung zeigt sich das Entwerfen als hochkomplexe Tätigkeit, auf die zahllose, zum Teil kategorial verschiedene Faktoren Einfluss nehmen: Wenngleich imaginativ und an individuelle Tätigkeiten gekoppelt, entwickelt sich der Entwurf in der Interaktion mit Werkzeugen, Medien oder Mitteln, die im Entstehenden ihre Spuren hinterlassen; ebenso können Ideen und Konzepte als Leitlinien große Wirksamkeit entfalten. Weiterhin spielt der soziale Austausch eine Rolle, in dem Teammitglieder, Klienten, externes Expertentum und die Interessen gesellschaftlicher Gruppierungen nicht unwesentlichen Einfluss ausüben und-nicht zu vergessen-lenken und leiten Behörden das 
Entstehende mit Genehmigungsverfahren durch Vorschriften und Gesetze, wie auch Normen und Richtlinien nicht folgenlos bleiben. Erlerntes und gemachte Erfahrungen bedingen das Ergebnis ebenso mit, wie es durch das kulturelle und soziale Umfeld geprägt wird.

Für eine Forschung, die ein besseres Verstehen der Entwurfsprozesse zum Ziel hat, stellt diese Vielfalt der Einflussfaktoren eine hohe methodologische Anforderung dar. Der vorliegende Band begreift Entwurfsforschung als eine genuin interdisziplinäre Aufgabe. Dadurch wird es einerseits möglich, einen Korpus heterogener Quellen zu erschließen-vom Archivmaterial über Entwurfstheorien zu Entwurfsmanifestationen - sowie andererseits unterschiedliche Verfahren anzuwenden, seien es Beobachtungen, Befragungen, explorative Tests oder Selbstreflexionen, seien es Beschreibungen, Begriffsklärungen, Analysen oder statistische Auswertungen. Eine übergreifende Entwurfsforschung lässt sich nicht auf den Gegenstand der Architektur beschränken, da Entwurfshandlungen auf vielen Gebieten stattfinden, beispielsweise im Designbereich, im Ingenieurwesen oder auch bei künstlerischen Verfahren. Hinzu kommt, dass das Erschaffen hybrider Artefakte und die damit einhergehenden Entwurfstechniken längst auch die Lebens- und Naturwissenschaften erreicht hat. Sicherlich mag die Diagnose eines »design turn « ${ }^{3}$ überspitzt sein, doch verweist der Ausdruck zu Recht auf die große Relevanz des Entwerfens. Die Entwurfsforschung im Rahmen dieses Bandes auf einen klassisch sentwerfenden (Gegenstand wie die Architektur einzugrenzen, hat dennoch zahlreiche Vorteile: Mit dem gemeinsamen Bezugspunkt treten nicht nur die Eigenarten der disziplinären Zugänge prägnant hervor, sondern es können Merkmale des Entwurfsprozesses dankbar herausgearbeitet werden. Architektonische Entwürfe werden in der Regel in Teams über einen längeren Zeitraum entwickelt. Das erfordert bestimmte Formen des Austauschs und der Kommunikation, die sich für eine Untersuchung anbieten; es liegen ausgereifte Notationsarten und differenzierte Werkzeugverwendungen vor und außerdem lassen architektonische Entwürfe komplexe Verflechtungen mit gesellschaftspolitischen und kulturellen Rahmenbedingungen erkennen. Auf diese Weise können am spezifischen Fall der Architektur wertvolle Einsichten gewonnen werden, die auf andere Bereiche und Disziplinen übertragbar sind.

\section{Felder der Entwurfsforschung}

Mit `Entwurf und `Entwerfen sind Kernbegriffe der Architektur benannt, die nicht nur zentrale Tätigkeiten ihrer Praxis 
beschreiben, sondern auch Angelpunkt der Reflexion und Selbstvergewisserung der Disziplin darstellen. Die Erforschung des Entwerfens innerhalb der Architektur (sowohl aus ihrem Tätigkeitsbereich heraus als auch in historisch-systematischer Hinsicht) ist nicht nur ein weites Feld, sondern, wie es Gerrit Confurius in den 1990er Jahren $^{4}$ einmal ausgedrückt hat, ein Feld, das durch einen »Hof von Unbestimmtheit" gekennzeichnet ist. Das ist nach Confurius kein Zufall, vielmehr Notwendigkeit, wenn sich »künstlerischer Geist« über »bloße Technik« erhebt. ${ }^{5}$ Davon abgesehen, dass die Trennung zwischen künstlerisch handelndem ingenium und bloßer technischer Umsetzung heute nicht mehr angemessen erscheint, lässt sich die Beschreibung des Themenkomplexes des Entwerfens als »unbestimmter Hof « bestätigen. In Hinblick auf unsere Ausgangsfrage, mit welchem Entwurfsbegriff die Architektur eigentlich operiere, würde dies bedeuten: mit so vielen Begriffen, wie es Entwerfende gibt.

In der Architektur wird die Hervorbringung von Ideen in Zeichnungen, werden Pläne, Modelle, sogar realisierte Gebäude als $>$ Entwurf bezeichnet. All das sind Forschungsgegenstände, aber auch andere Darstellungen des Entwerfens gelten als solche: etwa die seit Anbeginn der Architektur vorhandenen Selbstbeschreibungen von Arbeitspraxen und Ideenfindungsritualen, seien sie nun mehr als ,Wissenschaft oder mehr als handwerkliche oder künstlerische Traditionen entwickelt. Thematisiert wird dies bereits in der Traktatliteratur-von Urvätern wie Vitruv oder Alberti-und reicht bis hin zu den Entwurfsanleitungen oder -manifesten der Moderne, ${ }^{6}$ die wichtige Ausdrucksformen der Architektur bilden. Nicht die Geschichte oder die Geschichtstheorie haben diese Werke allein geschrieben, sondern zugleich die praktische Disziplin - wenngleich ihre Gegenstände später zu Quellen und Themen der Geschichtsund Theorieforschung geworden sind. ${ }^{7}$ Immanente Reflexionsprozesse und -aussagen der Architektur über ihre Entwurfsvorgänge sind in vielen Fällen für deren Praxis höchst einflussreich gewesen und sind es weiterhin; sie haben diese sforschend bereichert und wurden in der Folge immer wieder selbst zum Gegenstand der Forschung. Würden wir Äußerungen der Architekturschaffenden zu ihren Arbeiten ebenfalls berücksichtigen, wäre der Materialkorpus unüberschaubar. Er beschränkte sich dann nicht nur auf schriftliche Traktate und Manifeste, sondern müsste auch alle anderen Darlegungsformen umfassen (von der Baubeschreibung bis zur Kostenschätzung, von der Handskizze bis zum Haustechnikplan), denn sie 
alle haben den Entwurf zum Gegenstand. Dazu einen Überblick zu geben, ist hier weder möglich noch sinnvoll.

Eingedenk dieser »Unbestimmtheit« soll mit dem vorliegenden Band der Versuch unternommen werden, mehr Klarheit in die derzeitige Debatte zu bringen. Mit der nachstehenden Beschreibung einiger Felder, die im weitesten Sinne einer reflexiven Entwurfsforschung zuzurechnen sind und die allesamt ein besseres Verstehen von Entwurfsprozessen anstreben, sind bewusst Bereiche skizziert, in denen nicht nur die Disziplin der Architektur das Entwerfen erforscht. Sondern-und das ist gerade unser Anliegen-sie in einen Zusammenhang setzt mit der Wissenschaftsforschung, der Technikphilosophie oder den Kunst- und Kulturwissenschaften. Ein solcher Überblick muss zwangsläufig ausschnitthaft bleiben und unser Fokus auf die Literatur des deutschsprachigen Diskurses der letzten zwanzig Jahre wird vieles unerwähnt lassen.

Einen wichtigen Korpus der reflexiven Entwurfsforschung stellen Entwurfslehren der Architektur dar. Lehrkonzepte, beispielsweise als Lehrbücher verfasst, und damit verbunden auch die Entstehung von `Entwurfsschulen`, erweisen sich für die architekturhistorische Forschung als gut zugänglich. Sie können den Mangel an Quellen und Äußerungen, anhand derer sich nachvollziehen ließe, wie Architektinnen und Architekten konkret entwerfen, teilweise kompensieren. Teilweise deshalb, weil Aussagen zum eigenen Tun in der Architektur oft manifestartigen, postulierenden, sogar bewusst konstruierten Charakter besitzen, sodass diese als Zeugnisse zur Rekonstruktion von Entwurfspraktiken nicht immer geeignet sind oder zuerst mühsam aus heroisierenden Zuschreibungen herausgelöst werden müssen. ${ }^{8}$ Aufgrund der Breite des Genres seien hier nur wenige Beispiele genannt, die den Entwurfsunterricht aus historischer Perspektive untersuchen, wie etwa Klaus-Jürgen Winkler, Baulehre und Entwerfen am Bauhaus 1919-1933, oder die Studien zu dessen Nachfolgeschulen, die zur Versachlichung existierender Mythen beigetragen haben. ${ }^{9}$ Ähnliches gilt für Zeitphasen und Entwurfslehrkonzepte anderer Schulen, exemplarisch seien hier lediglich die Arbeiten genannt, die sich mit dem Status und der Außenwahrnehmung des Entwerfens an der ETH Zürich beschäftigen, ${ }^{10}$ oder der jüngst von Klaus Jan Philipp und Kerstin Renz herausgegebene Sammelband, der seinen Blick auf die Architekturschulen in Stuttgart, Dresden und Braunschweig richtet. ${ }^{11}$ Die Entwurfsforschung erweist sich als erneuernder Ansatz für architektur- und kunsthistorische Untersuchungen, vor allem dann, wenn sich retrospektiv 
Aussagen zu Prozessen der Formfindung nachvollziehen lassen. Ein Beispiel dafür ist der materialreiche von Ralph Johannes herausgegebene Band Entwerfen. ${ }^{12}$ Von Vitruv bis heute wird hier in zahlreichen Fallstudien dargelegt, wie die Ausbildung die Definition des Architektenberufs-und dessen sozialer Status auch die angewandten Entwurfslehren und das Entwurfshandeln-determinierten, wodurch letztlich deutlich wird, wie sich der Begriff des Entwerfens und seine Verwendung wandelte. Ein weiteres Beispiel für eine historische Herangehensweise liefert Frank Hassenewert, der in seiner Forschungsarbeit Lehren des Entwerfens ${ }^{13}$ den Diskurs anhand der Entwurfslehrbücher der zweiten Hälfte des 20. Jahrhunderts aufarbeitet.

Die Geschichtsforschung, die die an Schulen praktizierten ,Entwurfslehren net zum einen, wie über das Entwerfen im Sinne einer Veränderung, Verbesserung oder gänzlichen Neuausrichtung immer wieder nachgedacht wurde. Zum anderen zeigt sie aber auch, dass die dortigen Reflexionsprozesse für den gegenwärtigen Architekturunterricht relevant bleiben. So entstehen heute in der Entwurfslehre an Architekturschulen Dokumente, die entweder mehr in Berichtsform angelegt sind und einen deutlichen Anleitungscharakter tragen, oder solche, die kritisch-reflektierend auftreten. Hier überschneiden sich die Modi, was wiederum den Status des Faches in seiner Vieldisziplinarität abbildet. Methodische Grundlehren zum Entwerfen von Architektur werden laufend veröffentlicht, nicht zuletzt deswegen, weil das neue Bachelorstudium mehr Systematisierung, Modularisierung und Verdichtung der Inhalte erfordert. Ein Beispiel ist das "Cottbus Experiment«, in dem gezeigt wird, wie sich die BolognaReform und die Umstrukturierung des Architekturstudiums auf die Revision seiner Inhalte auswirkt. ${ }^{14}$ Die Zahl der (Ergebnis-)Berichte aus dem Entwurfsunterricht wächst und es seien hier nur zwei sehr unterschiedliche Beispiele aus dem Umfeld der ETH Zürich genannt: das Lehrprogramm des Lehrstuhls Dietmar Eberles, Von der Stadt zum Haus. Eine Entwurfslehre, das sich mit zentralen Rahmenthemen wie »Struktur", »Hülle« oder »Ort« beschäftigt; und die programmatischer angelegte Schrift des Lehrstuhls Marc Angélils mit dem Titel Deviations. Architektur Entwerfen, die antritt, um gewohnte Lehrsysteme zu hinterfragen. ${ }^{15}$ Ebenso versuchen Architektinnen und Architekten auch immer wieder mit der wissenschaftlichen Reflexion auf das Entwerfen ihr eigenes iTheoriegebäuder zu stützen. ${ }^{16}$

Das Themenfeld `Entwurfshandbücher ist eine wichtige Literatursparte des Faches Architektur, das von dem Anspruch 
geprägt ist, konzeptionelle Theoriegebäude, künstlerische Praxis und technische Umsetzung zu vereinen. Auch Handbuchmaterial entsteht in enger Verbindung mit der Praxis und ist sowohl Spiegel der Anwendung als auch Ausdrucksmittel der `Forschung`, denn es wird ebenso von den historischen und theoretischen Grundlagen der Architektur gespeist, wie es bei der Nutzung während des Entwerfens beständig überdacht und insofern sweitergeschrieben ‘ wird. Diese Literaturgattung wurde nicht erst im 19. Jahrhundert erfunden, hat zu diesem Zeitpunkt aber eine besonders reiche Ausbildung erfahren und zeigt die Ausdifferenzierung der Einzeldisziplinen, die sich in Bauprozessen zunehmend vollzog. Ihre Autorenschaft bilden zumeist Architekturschaffende. Die Beispiele sind zahllos, es soll hier nur auf zwei epochale und zueinander polare Werke verwiesen werden: Das eine ist das mehr als 140-bändige Handbuch der Architektur (1880-1943), das sowohl jeweils historische Grundlagen, als auch Beispiele für das Entwerfen aller möglicher Typen, Bauweisen und -arten enthält und allein in seiner Editionsgeschichte schon enzyklopädischen Anspruch entfaltet. ${ }^{17}$ Dagegen steht die modernisierte Version eines Handbuchs des Entwerfens, dessen Credo in der Verknappung eines immer komplexer werdenden Wissensfeldes und damit auch in einem Streben nach `Gesamtheit lag: die seit 1936 bis heute erfolgreich erscheinende Bauentwurfslehre von Ernst Neufert (derzeit 40. Auflage). ${ }^{18}$ In der ihr eigenen, kompakten Darstellungsform ist diese Entwurfslehre nicht die einzige, doch erreichte sie aufgrund der Nähe des Autors zur Baupraxis und einer schnell rezipierbaren Darstellungs- und Hand-habungsform einen Sonderstatus. ${ }^{19}$ Wie wichtig für die Erforschung dieser historischen Quellen auch die Beachtung von Fragen der Prozessualität und Medialität ist, zeigt etwa George Barnett Johnston. Er arbeitete zum amerikanischen Pendant der neufertschen Bauentwurfslehre, dem Architectural Graphic Standard, ${ }^{20}$ und macht in seiner Studie deutlich, dass über die `üblichen`Forschungsinstrumente der Architekturgeschichte hinaus spezifizierte Zugänge, etwa Methodiken der Sozialgeschichte oder der Diskursanalyse, notwendig sind.

Die Gattung der Entwurfshandbücher wird in ähnlicher Weise fortgeschrieben wie die Quellen zum Entwurfsunterricht. Es erscheint stetig, und meist vor dem Hintergrund einer langen Tradition, neue Anleitungsliteratur zum Holzbau, zum Glasbau, zum Dachbau, zum Fassadenbau und zu vielen anderen Teilbereichen der Architektur. Bücher dieser Art nennen sich gern »Atlanten«, die dann sowohl die Grundlagen des Faches als auch Anwendungsbeispiele 
und Detaillösungen für die Baupraxis beinhalten, und in einem hohen Durchdringungsgrad jeweils spezielle Bauteile oder -materialien sowie die begleitenden Entwurfsmöglichkeiten und -problematiken, aber weniger die Gesamtidee des Baus aufgreifen. ${ }^{21}$

Mit dem Anspruch informierend wie anleitend, kompilierend wie einführend zu sein, also Hand- und Lehrbuch gleichermaßen, ist zum Beispiel der Band für Architekturstudierende von Hanns M. Sauter, Arno Hartmann und Tarja Katz verfasst, Einführung in das Entwerfen, ${ }^{22}$ der sich darauf beruft, dass aus einer entwurfspragmatischen Haltung heraus die Anwenderinnen und Anwender immer wieder mit ähnlichen Fakten zu tun hätten, die sich deshalb über ein Handbuch standardisieren ließen. Wie fließend somit der Übergang zwischen Entwurfshandbüchern und Entwurfslehren ist, zeigen darüber hinaus auch jene Beispiele, die nicht aus den praktischen Bereichen der Architektur stammen, sondern die ihre `Entwurfslehre wiederum aus theoretischen Implikationen ableiten und so den Studierenden als intellektuelle Grundlage nützlich sein möchten. So weist Michael Bollé mit einer aus Werken der Architekturhistorie gespeisten Kompilation, dem Handbuch der Handbücher, ${ }^{23}$ darauf hin, den Studierenden sowohl Reflexion als auch Handlungsanleitungen zum Nutzen und Gebrauch mitzugebenkaum besser wird die Zwitterposition der Handbuch-Gattung damit deutlich gemacht. Davon abzusetzen sind Handbücher, die nach allen Richtungen überblickend, aber auch verkürzend geschrieben sind, so etwa die als $>$ Basiswerke $<$ verfassten Titel von Bert Bielefeld und Sebastian El Khouli, Entwurfsidee oder von Kari Jormakka, Dörte Kuhlmann und Oliver Schürer, Methoden der Formfindung. ${ }^{24}$

Anders als die Literatur der Handbücher, die Informationen für bestimmte Entwurfsfragen bereitstellen, versuchen Entwurfsmethodologien stärker in die Prozesse einzugreifen. Indem sie auf deren Verbesserung zielen, fließen hier deskriptive und präskriptive Elemente ineinander. Paradoxerweise ist die entwurfsmethodologische Forschung im deutschsprachigen Raum in der Architektur fast völlig zum Verschwinden gekommen-paradox insofern, als die Bewegung der 1960er Jahre zunächst deutlich von Protagonisten aus der Architektur geprägt war. Dazu mag beigetragen haben, dass sich einige der führenden Köpfe dieser Phase-wie beispielsweise Christopher Alexander, der mit Notes on the Synthesis of Form einen Klassiker geschrieben hat-im nachfolgenden Jahrzehnt vehement gegen eine Verwissenschaftlichung der Entwurfsforschung gewandt haben. ${ }^{25}$ Doch lässt sich aus diesem Umstand nicht hinreichend 
erklären, warum diese Forschungsrichtung in der Architektur so stark marginalisiert worden ist, denn andernorts und in anderen Disziplinen hat sich durchaus eine lebendige Tradition der Entwurfsmethodologie erhalten und weiterentwickelt. Im englischsprachigen Raum ist das interdisziplinär ausgerichtete Feld des Design Research fest etabliert, das im Architektur-Bereich mit Namen wie Bryan Lawson, Nigel Cross oder John Gero verbunden ist. ${ }^{26}$ Wenn auch im deutschsprachigen Raum Entwurfsmethodologie an den Architekturfakultäten kaum noch vertreten ist, findet sie Niederschlag in den Ingenieurwissenschaften, mit Klassikern wie der Konstruktionslehre von Gerhard Pahl, Wolfgang Beitz, Jörg Feldhusen und Karl-Heinrich Grote oder der angeleiteten Produktentwicklung, beispielsweise von Klaus Ehrlenspiel oder Udo Lindemann. ${ }^{27}$ Horst Rittel ist einer der wenigen Vertreter der Entwurfsmethodologie, dessen Schriften in der Architektur immer noch-oder vielleicht auch gerade wieder-zur Kenntnis genommen werden. ${ }^{28}$ Auffällig ist derzeit ein beginnendes, historisches Interesse, finden sich Vorboten einer Aufarbeitung in einigen, noch vereinzelt stehenden Veröffentlichungen. So untersucht beispielsweise Jesko Fezer die sich zuspitzende Krise der Bewegung Ende der 1960er Jahre; Gernot Weckherlin widmet sich den Tendenzen der Verwissenschaftlichung am Beispiel Nicholas Negropontes und Jürgen Joedickes; während Claudia Mareis-allerdings mit einem deutlichen Fokus auf den Design-Bereich-eine Diskursanalyse exemplarischer Positionen der Entwurfstheorie seit den 1960 er Jahren vornimmt. ${ }^{29}$

Es sind entwurfsmethodologische Arbeiten, auf die sich die Entwurfsforschung innerhalb der Technikphilosophie stützt und die sich insbesondere in der Diskussion um Heuristiken entfaltet hat. ${ }^{30}$ Hier sei verwiesen auf neuere Arbeiten, wie der von Pieter E. Vermaas, Peter Kroes, Andrew Light und Steven A. Moore herausgegebene Band, Philosophy and Design: From Engineering to Architecture, die explizit die Architektur als Gegenstand in die Betrachtung einbeziehen. ${ }^{31}$ Allerdings bleibt der Bezugspunkt der Fallanalysen in der Regel das Ingenieurwesen, insbesondere der Maschinenbau. Aufgrund der großen Ähnlichkeiten zwischen Entwurfshandlungen im Ingenieurwesen und in der Architektur dürften allerdings viele Befunde für beide Bereiche Gültigkeit besitzen. Bereits in den 1990er Jahren sind aus dem Umfeld der DFG-Forschergruppe Konstruktionshandeln eine Reihe von Veröffentlichungen hervorgegangen, die sich mit den Folgen des Computereinsatzes im Konstruktionsablauf auseinandergesetzt haben. ${ }^{32}$ Daneben sind es immer wieder 
interdisziplinäre Sammelbände, die Vorgänge des Entwickelns, Konstruierens und Entwerfens in den Mittelpunkt rücken, so etwa in Erkennen und Gestalten, in The Design of Material, Organism, and Minds, in Konstruieren zwischen Kunst und Wissenschaft oder Bedingungen und Triebkräfte technologischer Innovationen. ${ }^{33}$ Sie zeichnet aus, dass Positionen der Technikphilosophie und -geschichte in einen Dialog mit Vertreterinnen und Vertretern der gestaltenden Disziplinen treten. Das entstehende Wechselspiel zwischen spezifischen Studien in Teilbereichen der Architektur und des Ingenieurwesens und generellen Überlegungen macht deutlich, wie groß die Bandbreite von Entwurfsvorgängen und Entwurfsbegriffen ist-und wie vielfältig die Bezüge und Gemeinsamkeiten sind.

Wichtige Schnittstellen der technikphilosophischen Reflexion mit der Entwurfsforschung entstehen schließlich auch dort, wo zusätzlich medien- oder bildtheoretische Schwerpunkte aufgegriffen werden. So verweist Christoph Hubig auf den Doppelcharakter von Technik als Mittel und als Medium. ${ }^{34}$ Diese Bestandsaufnahme lässt Vorgänge der Invention und Innovation in einem neuen Licht erscheinen. Nicht nur die erzeugten Artefakte eröffnen strukturierte Möglichkeitsräume; das Gleiche muss für die technischen Geräte gelten, die im Entwurfsvorgang eingesetzt werden und dadurch die Bedingungen und Möglichkeiten des zu Entwickelnden entscheidend beeinflussen. Auch Sybille Krämer zeigt in ihren neueren Schriften, welch entscheidende operative Rolle bestimmten Formen von Bildern zukommen kann. ${ }^{35}$ Weit davon entfernt nur abzubilden oder zu visualisieren, kann mit deren Hilfe aktiv gearbeitet und exploriert werden, sodass sie das entstehende Artefakt mithervorbringen. Bilder werden somit zum »Werkzeug« und »Reflexionsinstrument«. Es sind gerade Studien dieser Art, die deutlich machen, dass sich die Diskussion über das Entwerfen vom Begriff des Disegno gelöst hat. ${ }^{36}$ Die Vorstellung einer Form gewordenen Idee, bei der sich die Imagination in der Zeichnung abbildet und der Entwurf als Zeichnung und als Idee vereint gedacht werden, lässt übersehen, wie wichtig der Einfluss materialer und medialer Bedingungen auf das Entstehende ist.

Werkzeuge und die mit ihnen verbundenen Praktiken stellen den zentralen Ansatzpunkt der Kulturtechnikforschung dar, die sich in den letzten Jahren dem Themenfeld des Entwerfens zugewandt hat. Dabei ist ein ganzer ’Kasten solcher Werkzeuge zu berücksichtigen, allerdings weniger in metaphorischer Ausdeutung, wie es etwa in Christian Gänshirts Studie, Werkzeuge für Ideen, angelegt ist. ${ }^{37}$ Sondern, es sind mehr die-wie im von Daniel Gethmann 
und Susanne Hauser herausgegebenen Band Kulturtechnik entwerfen genannten-Praktiken, Konzepte und Medien in Architektur und Design Science, die im ihren Fallstudien neue Perspektiven eröffnen. ${ }^{38}$ Kulturwissenschaftliche Erforschung des Entwerfens bedeutet hier, die Fixierung auf die Künstlerpersönlichkeit aufzugeben zugunsten einer Untersuchung von Materialien und Materialkulturen, Arbeitsbedingungen, Umfeldern, an Entwurfsprozessen beteiligten Agenten und Netzwerken. Deutlich wird damit artikuliert, dass von einem Entwurfsverständnis die Rede ist, das Schaffensvorgänge auch als methodisch zu erfassendes, zu analysierendes und als nachvollziehbar werdendes Tun versteht. Es sind Themen, die bereits in dem von Gundel Mattenklott und Friedrich Weltzien herausgegebenen Band Entwerfen und Entwurf anklingen, der Ansätze aus Praxis und Theorie verschiedener Schaffensprozesse in Kunst, Literatur und Musik zusammenführt. ${ }^{39}$

Gegenwärtig geht es nunmehr um eine Neubestimmung des Werkzeugbegriffs, deren sich vermehrt die Kulturtechnikforschung annimmt und die unter Entwurfswerkzeugen so Unterschiedliches versteht, wie etwa >Heuristikı, >Parametrikı und >Projektion`, ,Rekonstruktion` und ,Sammlung . Sie hat dabei nicht nur die Architektur und ihre klassischen Werkzeuge im Auge, sondern entleiht anderen Disziplinen Untersuchungsmethoden und Ergebnisse, die wieder in die Architektur zurückgespiegelt werden können. ${ }^{40}$ So untersucht etwa die vierbändige, von Christoph Hoffmann und Barbara Wittmann herausgegebene Reihe Wissen im Entwurf auf den Spuren der Wissens- und Wissenschaftsgeschichte entwerfende Handlungen in unterschiedlichen Bereichen. ${ }^{41}$ Charakteristisch für dieses Forschungsfeld ist, dass sowohl in seinem Entwurfs- als auch in seinem Werkzeugbegriff das gegenseitige Bedingungsverhältnis aller Agierenden in Entwurfsprozessen anerkannt ist. ${ }^{42}$ Entwerfen ist dabei auch im Sinne »rekursiver Operationsketten $\aleph^{43} \mathrm{zu}$ denken. Wenn eine solche Kulturtechnikforschung eine historische Betrachtungsweise des Entwurfs vornimmt, dann ist sie eben nicht nur auf das Subjekt bezogen, sondern ebenso auf die Werkzeuge und auf die Medien. Dies eröffnet neue Blickwinkel, die von der Architektur aufgegriffen werden können, um Fragen wie die nach dem »Machen der Architektur ${ }^{44}$ aus einer anderen Perspektive zu stellen.

Ein die Entwurfsforschung erneuernder Anstoß kommt auch aus der Architekturgeschichte, wenn diese mehr und mehr die gründliche Betrachtung-beispielsweise der Instrumente und der Verfahren-vornimmt, wie dies seit den letzten zwei Jahrzehnten 
eine Reihe von Veröffentlichungen zu zeichnerischen Notationen, zu Projektionsverfahren oder zum Modellgebrauch zeigen. Die mediale Vielschichtigkeit der Architekturzeichnung als technischkonkretisierendes und auch darstellendes Entwurfsmittel wurde in seiner historischen Ausprägung bereits mit dem Ausstellungskatalog Winfried Nerdingers, Die Architekturzeichnung, angesprochen; ${ }^{45}$ bis heute hat sich diese Forschung zugunsten von Fragen nach Operationalität, nach konkret angewandten Instrumenten und nach expliziten Verfahren verschoben. Dies verdeutlicht etwa der von Susan C. Piedmont-Palladino herausgegebene kleine Band, Tools of Imagination, der Zeichenwerkzeuge als wesentlich zu beachtende Instrumente der Ideenproduktion hervorhebt. ${ }^{46}$ Standardwerke $\mathrm{zu}$ historischen Fragen der Projektion sind etwa Robert Evans, The Projective Cast, das den Status von Mathematik und Geometrie, die Zeichnung in technischen wie ästhetisch bestimmten Entwurfsprozessen, die Übertragungstechnik der Projektion (einschließlich deren Metaphern und Ambiguitäten) und der daraus resultierenden Bildlichkeit der Architektur seit dem 15. Jahrhundert untersucht. ${ }^{47}$ Ebenso ist die umfassende Studie von Alberto Pérez-Gómez und Louise Pelletier, Architectural Representation and the Perspective Hinge, $\mathrm{zu}$ nennen, die Seh-, Übertragungs- und Transformationsprozesse seit dem 17. Jahrhundert darstellt, die sich weitgehend unter dem Begriff des »Perspektivischen « erörtern lassen und die entwurfsdeterminierend wirkten. ${ }^{48}$

Als einer der Repräsentanten des Entwurfs steht auch das >Modell als Artefakt und als Begriff seit längerer Zeit unter Beobachtung-zunächst sind es die eindrucksvollen Renaissance-Modelle, ${ }^{49}$ die Gegenstand der Forschung wurden; aber auch Werner Oechslin hat das historische Modell jüngst wieder aufgegriffen und unter dem Stichwort »Idea materialis« als eines der Medien der Architektur (bzw. des Architekturentwerfens) beschrieben. ${ }^{50}$ In dem Sammelband von Marco Frascari, Jonathan Hale und Bradley Starkey, From Models to Drawings, ${ }^{51}$ werden hingegen beide Repräsentationsformen, die Zeichnung und das Modell, in einen näheren Zusammenhang gebracht, deren historische Perspektiven, Fähigkeiten zur Realitätserzeugung und auch deren kritische Dimensionen untersucht. Welche Bedeutung die Frage der Repräsentation in der Architekturgeschichte generell zufällt, ist spätestens seit Mario Carpos Buch Architecture in the Age of Printing bekannt, das den Fall Alberti unter einer neuen Lesart behandelt. Der Fakt der "Entwurfsstandardisierung« ist hier nicht von den Mitteln und Wegen medialer Vervielfältigung zu 
trennen. Davon ausgehend proklamiert Carpo in seiner neuesten Studie zu algorithmischen und parametrischen Entwurfsszenarien eine neue unendliche Variabilität und damit auch `Kunsthandwerklichkeit< des Entwerfens. ${ }^{52}$

Die Betonung der Praxis, ihrer Werkzeuge und Techniken finden sich auch in Studien aus dem Bereich der Wissenschaftsforschung wieder. Angeregt durch die frühen Laborstudien in den Naturwissenschaften, beispielsweise von Bruno Latour, Steve Woolgar oder Karin Knorr Cetina, wenden sich neuere Arbeiten mit ihrem ethnografischen oder mikrosoziologischen Interesse zunehmend auch kreativen Prozessen zu. Die Architekturstudien sind gekennzeichnet durch minuziöse Beobachtungen direkt vor Ort in den Büros, welche über einen längeren Zeitraum (von einigen Monaten bis zu mehreren Jahren) begleitet, und die in der Regel um Tiefeninterviews mit den Beteiligten ergänzt werden. Auffällig ist, dass neben sprachlichen Dokumenten zugleich Zeichnungen, Modelle, Instrumente oder Gesten Bestandteile der Analyse sind. Zu den sehr frühen Studien gehört die Untersuchung Architecture. The Story of Practice von Dana Cuff, die die komplexen Handlungen auf der Suche nach tragfähigen Entwurfslösungen anhand konkreter Beispiele aus der Praxis nachzeichnet. ${ }^{53}$ Meist spielt die Frage nach der Wissensgenese eine wichtige Rolle, wenngleich die Studien auch unterschiedliche Schwerpunkte ausbilden: Beispielsweise verfolgt Jürgen Potthast eine Hochhausplanung in Berlin Ende der 1990er Jahre unter besonderer Berücksichtigung der dabei eingesetzten Kreativitätstechniken; Albena Yaneva untersucht am Beispiel einer Projektgruppe bei OMA vorrangig die Rolle von Modellen; Sophie Houdart analysiert die Funktionen von Renderings als Textverfahren am Beispiel einer Entwurfsplanung im Büro von Kuma Kengo; Keith Murphy beschreibt den Einsatz von Gesten, Werkzeugen und Zeichnungen als Mittel kollektiver Imagination in Entwurfsprozessen; während Boris Ewenstein und Jennifer Whyte der Frage nachgehen, inwiefern sich Entwurfsprozesse als Wissenspraktiken klassifizieren lassen. ${ }^{54}$ Durch eine neutrale Haltung in der teilnehmenden Beobachtung, die in der Regel durch fachfremde, aber an der Architektur interessierte Laien durchgeführt werden, gelingen Darstellungen des Entwurfsprozesses, die ohne jene Klischees oder Präjudizierungen auskommen, die dem Entwurf angelastet werden. Es ist gerade dieser unbedarfte Blick, der gern übersehene Aspekte des Entwurfsprozesses verdeutlichen kann-anders als es ein innerdisziplinärer Blick je vermöchte. 


\section{Zu den Beiträgen dieses Bandes}

Der vorliegende Sammelband bündelt eine Reihe neuer Forschungsbeiträge, die sich aus verschiedenen Perspektiven mit dem Spannungsverhältnis zwischen Entwerfen, Wissenschaft und Forschen auseinandersetzen. In ihrer Bandbreite loten sie zugleich das Spektrum einer reflexiven Entwurfsforschung aus, die auf ein besseres Verstehen des Entwerfens zielt und sich ihrer analysierenden Metaebene bewusst ist. Wie die obige Skizze einzelner Felder der Entwurfsforschung gezeigt hat, lässt sich eine klare Unterscheidung zwischen Quellen und Literatur der Entwurfsforschung oftmals nicht vornehmen. Was in einem Fall bereits eine Theoriebildung über Entwurfsvorgänge darstellt, kann in einem anderen Fall-etwa bei historisch oder systematisch orientierten Arbeiten - zu einer Primärquelle für die Entwurfsforschung werden. Auffällig ist zudem die Fülle an Materialien, die kaum heterogener sein könnte und ebenso Fremd- wie Selbstbeobachtungen, Texte wie Bilder, historische Vermächtnisse wie gegenwärtige Provisorien, umfasst-denn es sind gerade jene ephemeren Manifestationen, die den Entwurfsvorgang bestimmen und selten lange Zeit überdauern. Die Prozessualität aber, stellt traditionell mit geisteswissenschaftlichem Instrumentarium arbeitende Bereiche vor neue Herausforderungen - ganz anders als eine an Gegenständen und Resultaten ausgerichtete Forschung. Auf der Suche nach Lösungen, ist es nicht zuletzt der Überblick der Beiträge, der die disziplinären Zugangsweisen in einen spannungsvollen Dialog bringt. Wenn er gelingt, können aus der Verschränkung neue Wege hervorgehen, um die reflexive Entwurfsforschung als eigenständiges Forschungsfeld zu etablieren.

Vier aufeinander aufbauende Rahmenthemen, die untereinander vielfältige Bezüge aufweisen, gliedern den vorliegenden Band. Der erste Abschnitt »Entwerfen als Gegenstand der Forschung " untersucht, wie eine Forschung über das Entwerfen angelegt sein kann. Die dabei sichtbar werdende Bandbreite von historischen über systematischen bis zu empirischen Zugängen steht für das Spektrum einer reflexiven Entwurfsforschung, die in der Wahl ihrer Methoden und Theoriemodelle selbst zum Exempel wird. Aus architekturhistorischer Perspektive fragt der Beitrag »Jäger, Sammler, Architekten. Oder, wie «kolligierende Prozeduren des architektonischen Entwerfens beschrieben und erforscht werden können« von Eva Maria Froschauer, wie das Sammeln als sWerkzeug` des Entwerfens konturiert werden kann. Es wird gezeigt, wie das konstituierende Moment des Sammelns und das kreierende des Entwerfens in der 
Architektur nicht nur zusammenwirken, sondern auch gemeinsam erforscht werden können. Dabei wird zunächst den Fragen nachgegangen, warum Sammeln als eine Prozedur zu verstehen ist, die vielen Entwurfsvorgängen inhärent ist und sich als Entwurfswerkzeug beschreiben lässt. Anschließend wird skizziert, wo und in welcher Form das Sammeln innerhalb der Architekturgeschichte als Forschungsthema historisch wie systematisch zu verorten ist. Im dritten Schritt sind verschiedene Herangehensweisen einer solchen Untersuchung skizziert-von der Sammlerseite aus, vom Objekt her oder von verschiedenen Anwendungsbezügen aus denkend. Als Fazit werden die Großbegriffe `Sammeln und `Entwerfen kus eines, Wissenswegs zusammenführend betrachtet.

Aus der Ethnologie kommend widmet sich Ignacio Farías in seinem Beitrag »Epistemische Dissonanz. Zur Vervielfältigung von Entwurfsalternativen in der Architektur« dem spraktischen Problem des Erkenntnisgewinns im Entwerfen, das sich beständig mit dem Generieren von noch nicht Existierendem, von Neuem beschäftigt. Er wendet hierfür ein ethnografisches Instrumentarium an, um seine Betrachtungen auf ein »Wissen-in-Aktion« zu richten. Seine These, die er mit der Feldforschung in drei Architekturbüros bestätigt sieht, besagt, dass Fortschritt in Entwurfsprozessen wesentlich durch die Entwicklung von Designvarianten vorangetrieben wird, die erst durch ihre »epistemische Dissonanz« möglich werden. Farías schließt nach dem von David Stark geprägten Begriff der »evaluativen Dissonanz«, dass die Menge unterschiedlicher Standpunkte und Kenntnisse, wie sie im Arbeiten der Architekten zutage treten, eben dieses Epistem der Dissonanz formt. Der Autor zeigt in diesem Zusammenhang auch, welches hohe Potenzial etwa die sogenannten Korrektur-Termine, die zwischen allen, am Projekt beteiligten Architekturschaffenden laufend stattfinden, zur Mobilisierung des Wissensbildungsprozesses im Entwerfen beitragen.

Aus kulturwissenschaftlicher Perspektive zeigt der Aufsatz von Liliana Gómez, »Entwerfen als Wissensorganisation. Kulturelle Transformationsprozesse im Spiegel der amerikanischen Architektur der 1950er und 1960er Jahre«, wie während der Zeit des Kalten Krieges Architektur entworfen wurde und auch selbst entworfen hat. Gómez begreift, den Thesen Reinhold Martins folgend, Architektur als Medium und stellt dar, wie deren Entwerfen unter den damals herrschenden Verflechtungen von Industrie-, Militär- und Forschungsinteressen als Technik einer Organisation von Wissen zu verstehen ist. Anhand der corporate architecture, der Architektursprache 
großer Industriekonzerne, wie etwa Eero Saarinens Entwürfe für IBM oder Richard Buckminster Fullers Dymaxion-Projekt, weist die Autorin nach, wie vor dem Hintergrund kultureller Wandlungender kybernetischen Wende und der heraufdämmernden Durchdringung der Gesellschaft durch den Computer-Übertragungsprozesse im Entwerfen stattfanden bzw. sich abbildeten.

Aus Sicht der Philosophie beschließt Hans Poser mit seinem Beitrag "Ars inveniendi heute. Perspektiven einer Entwurfswissenschaft der Architektur « diesen ersten Teil des Bandes. Auf den Spuren der ars inveniendi, der »Kunst des Erfindens«, einem Begriff aus der Rhetorik, analysiert der Autor das Problem der Verwissenschaftlichung der Kunst des Erfindens und Entwerfens bis in die Gegenwart. Ausgehend von den leibnitzschen Darlegungen einer ars inveniendi nimmt Poser kritisch in den Blick, ob, und wenn ja, wie das Entwerfen als eine Wissenschaft verstanden, methodisch erfasst und in einem »Möglichkeitsdenken" verortet werden kann. Damit ist die grundsätzliche Frage aufgeworfen, ob etwas, was Kreativität erfordert, überhaupt in eine Wissenschaft überführt werden kann. Hier sind deutliche Grenzen jeder Methodenlehre aufgezeigt, so der Autor weiter. Eine Entwurfswissenschaft kann etwas anderes leisten, indem sie bestimmte Wissensformen zur Verfügung stellt, die ein Problemlösungswissen ausmachen, und zugleich gekoppelt ist an eine Offenheit für Neues als zwingende Voraussetzung.

Der zweite Abschnitt des vorliegenden Sammelbandes, »Verwissenschaftlichung des Entwerfens«, widmet sich der Frage, inwieweit Entwurfsprozesse selbst einer stärkeren Verwissenschaftlichung zugänglich gemacht werden können, welche Konsequenzen daraus für die Entwurfshandlungen folgen und wie eine Wissenschaft des Entwerfens zu fassen sei. In dem ersten Beitrag dieses Abschnitts mit dem Titel »Vom Betriebscharakter des Entwerfens. Konjunkturen der Verwissenschaftlichung in der Architektur« zeichnet Gernot Weckherlin an einigen historischen Fallbeispielen die wechselnde Hinwendung zur Verwissenschaftlichung architektonischer Entwurfsprozesse nach. Die gegenwärtig wieder gern vorgebrachte Forderung nach Wissenschaftlichkeit und Objektivierung, die auch in der Architekturausbildung an Hochschulen erhoben wird, sei, so die These des Autors, längst ähnlich in Martin Heideggers Beobachtungen zum »Entwurfscharakter« der Wissenschaft als Forschung abzulesen gewesen und deshalb auch zu relativieren. Trotzdem nimmt der Autor den behaupteten »Betriebscharakter« zum Ausgangspunkt seiner Ausführungen, um kritisch verschiedene 
Verwissenschaftlichungstendenzen in der Geschichte des Architekturentwerfens zu vergleichen. Er tut dies entlang veröffentlichter Schriften gebauter Beispiele in chronologischer Reihung von William Richard Lethaby zu Hannes Meyer, von Ernst Neufert zu Horst Rittel, um dann mit Meinhard von Gerkan von einem gegenteiligen Fall zu berichten, für den dieser konstatierte, dass Verwissenschaftlichung doch nur eine Tendenz zur unglücklichen »Theoretisierung" der Architektur sei.

Wie bei einem Beispiel der Gegenwartsarchitektur die Neuordnung der Instanzen Entwurf, Werkzeug, Maschine, Medium und die künstlerische Tätigkeit selbst unter dem Einfluss der Operationalisierung aussehen kann, zeigt Nathalie Bredella in ihrem Beitrag »Modelle des Entwerfens. Zur Bedeutung digitaler Werkzeuge im Entwurfsprozess von Frank O. Gehry«. Die Autorin weist nach-mithilfe der genauen Beschreibung von Einsatz und Wirkungsweise digitaler Werkzeuge-, wie ralter und sneue Medien in der Entwurfsarbeit zusammenwirken können, welche operativen und personellen Veränderungen sich dadurch ergeben und wie sich diese auf das Entwurfsergebnis auswirken. Sie analysiert dazu drei Projekte des Büros Gehry Partners (Los Angeles) aus den 1990er Jahren: den Barcelona Fish, das Guggenheim Museum in Bilbao und die Walt Disney Concert Hall in Los Angeles. Anhand der Beispiele wird deutlich, wie durch digitale Modelle, neue Software-Lösungen und vor allem auch durch die noch immer zum Einsatz kommenden analogen Verfahren, die gesamten Rahmenbedingungen des Entwerfens neu geordnet werden. Auf dieser Grundlage ergibt sich, so Bredella, auch eine Neubewertung der Bedeutung digitaler Technologien im Rahmen der Entwurfsprozesse.

Aus Sicht der Bauinformatik untersucht Philipp Geyer in seinem Beitrag »Von der digitalen Linie zum Systemmodell. Information und Wissen in der Entwurfsmodellierung mit dem Computer«, wie sich digitale Modelle in den letzten zwanzig Jahren verändert haben. In einem systematisch-historischen Überblick zeichnet er die Entwicklung vom digitalen Zeichnungsmodell über die Modellierung mit bauwesenspezifischen Objekten bis zu verschiedenen Arten der parametrischen Modellierung nach. Exemplarisch für die sich derzeit noch in der Forschung befindliche, an das Systems Engineering angelehnte Systemmodellierung wird deren Anwendung in Hinsicht auf Energieverbrauch und Umsetzung von Nachhaltigkeitskriterien in Entwurfsprozessen diskutiert. Abschließend stellt der Autor die Frage, welche Rolle Information und Wissen in diesen 
Modellen übernehmen. Eindeutig erkennbar ist die Zunahme ihrer Einbettung in Informationssystemen, was als Hinweis für eine Tendenz der Verwissenschaftlichung gewertet werden kann.

Zum Abschluss dieses Abschnitts erarbeitet Christoph Hubig aus Sicht der Technikphilosophie in seinem Beitrag »Dialektik des Entwerfens. Entwurfswissenschaft als Reflexion", wie sich eine reflektierende Entwurfswissenschaft aus der dem Entwerfen immanenten Dialektik verstehen lässt. Der Autor geht davon aus, dass Entwurfsbeschreibungen meist in Dichotomien angesiedelt sind, das Entwerfen in sogenannten "Spannungsfeldern", "Zwischenreichen" oder "Beziehungsräumen« verortet wird und somit polare Beziehungspaare wie "Kunst und Technik", »Intellekt und Intuition « oder »Methodik und Kreativität« aufgerufen werden. Hubig geht es darum, solche »Binnenbeziehungen « systematisch zu betrachten und das Problem unter dem, wie er schreibt, weit »strengeren« Konzept einer hegelschen Dialektik zu untersuchen. Der Beitrag geht hierfür in drei Stufen vor: vom »An-Sich« des Entwerfens, das als »reale Möglichkeit«, als »Horizont« oder als »Vorgabe« erscheint, zum "Für-Sich«, welches als »bestimmte Wirklichkeit«, als »Begrenzung", als "Ausschluss« gesehen werden kann, hin zum "An-und-Für-Sich», wo sich die Entwurfswissenschaft selbst formiert.

Der dritte Abschnitt dieses Sammelbandes, »Entwerfen als Mittel der Forschung «, hinterfragt kritisch, ob der Entwurfsvorgang selbst zum wissenschaftlichen Verfahren werden kann, wo eine Nähe zu Vorgehensweisen naturwissenschaftlicher Disziplinen liegt, aber auch danach, wo signifikante Unterschiede festzustellen sind. Philip Ursprung zeigt in seinem Beitrag »Exponierte Experimente. Herzog \& de Meurons Modelle« welchen Prozeduren das Entwerfen des seit den ausgehenden 1970er Jahren weltweit arbeitenden Basler Architekturbüros folgt. Innerhalb der arbeitsteiligen Prozesse des Büros kann analysiert werden, wie Entwurfsideen, -hypothesen und -ergebnisse in einem großen Team weitergereicht werden und wie der Wissensbildungsprozess funktioniert. Doch stellt sich dabei die Frage, ob die »Experimentalanordnungen" dieser Architekten als ,forschend bezeichnet werden können-in dem Sinne, dass die Ausgangssituation transparent und die Arbeitsschritte nachvollziehbar sind. Denn letztlich geht es im Entwerfen Herzog \& de Meurons immer um die Innovation der Prozeduren und folglich um die Entwicklung einer, jeweils der einzigartigen Situation genügenden, spezifisch >künstlerischen Antwort. So zeigt Ursprung anhand mehrerer Projekte und besonders mit dem Entwurf von Prada Tokyo und den dabei 
verwendeten zahlreichen Konzeptmodellen, dass das Entwurfsprozedere an vielen Punkten zwar quasi wissenschaftlich nachvollziehbar bleibt (auch aufgrund der handwerklichen Herstellung der Modelle), aber letztlich als künstlerisches Paradigma zu sehen ist.

In der Traditionslinie, die in Architektur und Planung das Handeln und Vorgehen der Entwerfenden als rational beschreibbar versteht, liegt die Schwierigkeit, dass in späteren Untersuchungen rationales Handeln aus künstlerischen Prozessen schwer herauszufiltern ist. Henrik Hilbig begegnet diesem Problem in seinem Beitrag »Was Fahrradfahren, wissenschaftliche Erkenntnis und ein Hotelentwurf miteinander zu tun haben. Ein Versuch mit Michael Polanyi«. Er bietet eine Lösung an, indem er zwei Ebenen zusammenführt: Auf der einen diskutiert er Polanyis Begriff Tacit Knowledge, der sich als Konzept dem sogenannten stillen oder impliziten Wissen widmet; auf der anderen Ebene der Selbstbeschreibung analysiert der Autor den Entstehungsprozess eines Hotelentwurfs. Hilbig kann auf diese Weise zeigen, dass Entwerfen sich nicht unbedingt als ein rationaler Prozess rund um die Beantwortung einer genauen Ausgangsfrage erweisen muss. Stattdessen offenbart sich, dass das polanyische Konzept-dass wir mehr wissen, als das, was wir zu sagen vermögen-in Entwurfsprozessen gespiegelt sein kann. Nämlich genau dort, wo der Beginn des Prozesses den Ausgang offen lässt.

Der Frage, inwiefern sich Entwerfen als Mittel der Forschung verstehen lässt, nähert sich der Beitrag "Wie Architektur entsteht. Entwerfen als epistemische Praxis« von Sabine Ammon auf indirekte Weise. Exemplarisch diskutiert sie mithilfe einer ethnografischen Untersuchung von Albena Yaneva die derzeit vielfach geäußerte Behauptung vom Entwerfen als Experimentieren. Dabei arbeitet Ammon heraus, dass - trotz einiger Gemeinsamkeiten - sich das architektonische Entwerfen und Experimentieren aus erkenntnis- und wissenschaftstheoretischer Sicht deutlich unterscheiden. Anhand einer Skizze der im Entwurfsprozess epistemisch wirksam werdenden Techniken, Werkzeuge und Strategien kann sie aufzeigen, dass mit dem Entwerfen eine eigenständige epistemische Praxis vorliegt, die ein Wissen über das Entworfene hervorbringt, das jedoch noch nicht als wissenschaftliches Wissen zählen kann.

Mit Susanne Hausers Aufsatz »Verfahren des Überschreitens. Entwerfen als Kulturtechnik« schließt dieser dritte Buchabschnitt. Eine zentrale Fähigkeit von Entwurfsprozessen-nämlich das `Modellieren $<$-kann sich in jedem Einzelschritt mit der Entstehung von Unerwartetem zeigen. Im Modellieren neuer Gegenstände 
und Ideen überschreitet somit jede gefundene Gestaltungsform das Bisherige. Die Autorin untersucht auf Basis dieser Feststellung, wo und wie genau sich solche ,Überschreitungen zeigen, welche Potenziale sie jeweils bergen und in welchen Praktiken sie sich manifestieren. Hauser begibt sich dabei auf die Metaebene, indem sie fragt, wie diese Vorgänge bislang erforscht wurden und wie dies künftig geschehen könnte. Mit diesen beiden Schwerpunkten entwickelt der Beitrag eine aufbauende Dramaturgie, indem vom Begriff der "Modellierung« zu jenem des »Neuen« und »Künftigen« geschlossen wird und somit das produktive Potenzial der Kulturtechnik Entwerfen noch einmal umrissen ist. Danach werden deren »Praktiken und Methoden« benannt sowie die entstehenden »Paradigmata«; trotz der großen "Fragilität des Zusammenhangs«, so schließt die Autorin weiter, leistet das Entwerfen eine "Synthese«, die am Ende der Vorgänge zu »Stimmigkeit» führen soll.

Der vierte und letzte Abschnitt des Bandes, "Wissenschaftliche Vermittlung des Entwerfens«, zeigt an historischen und aktuellen Beispielen, wie das Entwerfen gelehrt und vermittelt werden kann. In dem Maße, in dem die Architektur sich darum bemüht, ihre Entwurfslehren zu systematisieren, sucht sie das gewonnene Wissen auch festzuschreiben und zu lehren. Entwurfslehren haben also immer auch Einfluss auf die jeweiligen Tendenzen der Verwissenschaftlichung des Entwerfens, ebenso wie auf die akademische Ausdifferenzierung des Fächerspektrums an Hochschulen und Universitäten. So untersucht die Kunsthistorikerin Christiane Salge in dem Beitrag »Ästhetik versus Wissenschaft. Die Entwurfsausbildung an der Bauakademie in Berlin (um 1800) « die Erneuerung der Architektenausbildung in Preußen. Was in dem damaligen Reformprozess substanziell diskutiert wird und bis heute als Fragestellung virulent geblieben ist, ist die Verhältnisbestimmung von künstlerischen Inhalten $\mathrm{zu}$ »wissenschaftlich « gesicherten Erkenntnissen im Unterricht einer solchen Ausbildungsstätte. Die Debatte dieser Zeit verändert die Entwurfsausbildung in der Architektur nachhaltig, indem neue Wissensbestände für die Lehre erschlossen werden. Das Kopieren von Vorlagen oder das Zeichnen von Modellen wird abgelöst durch die Vermittlung systematischer Grundkenntnisse. Zum Bestandteil des Curriculums werden neben Baukunstgeschichte oder Bautypologien »wissenschaftliche Fächer«: Gelehrt werden nun ebenso Konstruktion, Physik, Mechanik, Optik oder Perspektivisches Zeichnen. Obgleich das Fach Entwerfen damals als Terminus noch nicht ausgebildet ist, kann die Autorin mit der Auswertung 
von Archivalien, von Lehrplänen und -büchern rekonstruieren, wie der »Entwurfsunterricht» an der Berliner Bauakademie ausgesehen haben muss.

Was die Architekturtheorie aus der Betrachtung der Entwurfslehren der Maschinenkonstruktion lernen kann, erläutert Wolfgang König in seinem Beitrag »Zwischen Algorithmus und Intuition. Ein Analogieangebot für die Architekturtheorie aus der Geschichte der Maschinenkonstruktion«. Er stellt dabei den Positionen zweier Konstruktionswissenschaftler aus dem 19. Jahrhundert, jene Franz Reuleaux' und Alois Riedlers, mit Fritz Kesselring und Johannes Müller zwei Positionen aus dem 20. Jahrhundert vergleichend gegenüber. Bei jeweils zwei der Protagonisten sieht König einen eher intuitiven Ansatz, also ein empirisches Vorgehen, und einen theoriebasierten Ansatz in Form einer Konstruktionslehre, bei dem Algorithmen eine große Rolle spielen. Allerdings lässt sich keine Entwicklung von einer erfahrungsbasierten zu einer theorielastigen Entwurfsrichtung feststellen. Im Gegenteil, es existieren beide Schulen mit ihren diversen Vor- und Nachteilen nebeneinander und repräsentieren auf diese Weise die nicht aufzuhebende Spannung zwischen Praxis und Theorie. Dieses, so König, scheint im gleichen Maß auch für die Architektur zu gelten, womit er die Sichtweise deutlich in Zweifel zieht, die in der fortschreitenden Formalisierung des Entwerfens eine Art swissenschaftliches Allheilmittel sieht.

Unter dem Titel »Homologie-Experiment. Ein Versuch, das Entwurfspotenzial von Werken der Architektur für die Lehre verfügbar zu machen « betrachtet der Architekt und Hochschullehrer Alban Janson das Lehrfach Architekturentwurf als ein Experimentierfeld. Er geht davon aus, dass die Geschichte der Architektur reichlich Grundlagen und damit vor allem ein »strukturelles Repertoire« zur Verfügung stellt, das sowohl für den Entwurfsvorgang selbst als auch für die Vermittlung des Entwerfens genutzt werden kann. Allerdings soll es aus vorhandenen und nützlichen Strukturen, aus realen und historischen Objekten »herausgelesen « und für die Gegenwart und das Entwerfen verfügbar gemacht werden. In Anlehnung an Roland Barthes muss dies, so Janson, im Sinne eines strukturalistischen Arbeitens geschehen, wobei er sich auf dessen Text Die strukturalistische Tätigkeit bezieht. Dies dürfe aber nicht einem einfachen Analogieschluss folgen, sondern als »Homologie« müsse gelehrt und gelernt werden, wie Strukturen gedeutet und interpretiert werden können. Jansons Beitrag zeigt am Beispiel von Studienarbeiten und einem gegenständlichen Lehrinstrument, einer 
kleinen »kinetischen Apparatur", wie eine solche strukturalistische Tätigkeit und damit eine Entwurfsmethode in der Architektur vermittelt werden kann.

Der Beitrag der Architektin Judith Reeh, ")Grundlehre Entwerfen. Ausbildungspraktiken an den TU9-Universitäten in Deutschland", vergleicht den Einführungsunterricht in das Entwerfen, wie er an zwei technischen Universitäten, in Aachen und in Berlin, praktiziert wird. Anhand konkreter Beispiele zeigt sie, wie dort jeweils am Beginn des Studiums Übungsreihen zum Entwerfen aussehen, damit dieser erste "Aufbauprozess" des "entwurflichen Wissens" gelingen kann. Reehs Beitrag schließt die Analyse des Lehrmaterials, der strukturierten Übungsaufbauten, der verschiedenen Lehrinstrumente, -formate und -hierarchien der Lehrenden ein, die auf diese Weise verglichen werden können. Gerade die Grundlehre Entwerfen eignet sich gut für eine solche Untersuchung, da im ersten Studienjahr zunächst in die wichtigsten Wissensfelder des Entwerfens eingeführt wird und dabei Techniken und Regeln zur Konzeptentwicklung unterrichtet und die Möglichkeiten der visuellen und materiellen Repräsentation vermittelt werden. All dies gelingt in der notwendigen Verdichtung nur mit schlüssigen wie auch flexiblen Lehrkonzepten. Womit gezeigt ist, dass die volatilen Prozesse des Entwerfens selbst beständig das Lehren, die Vermittlung und letztlich auch deren Reflexion beeinflussen-eine Einsicht, die nicht nur am Ende dieses Abschnitts steht, sondern auch den Sammelband beschließt. 